\section{Direct Methods of Phase Determination}

MS02.03.01 STRUCTURE DETERMINATION OF HELICAL AND HAIRPIN PEPTDES WITH UPTO 30 RESIDUES PER ASYMMETRIC UNIT. Isabella L. Karle, Laboratory for the Structure of Matter, Naval Research Laboratory, Washington, D. C. 20375-5341, USA

Practical information for the successful application of vector search/translation function/tangent formula expansion using the known structure of a fragment from another crystal to derive the structures of molecules containing 100 to $300 \mathrm{C}, \mathrm{N}$ and $\mathrm{O}$ atoms will be presented. Large molecules containing $S$ or heavier atoms will not be cliscussed since the heavier atoms usually significantly facilitate the structure solution by the Patterson function, direct phase determination or anomalous dispersion differences (see the 308 non-H atom structure, Konnert, Karle and Karle, Montreal ACA Abstracts 23, 2m6 A (1995)). The structure solutions of the following will be described: $\beta$-turn/ $\beta$-sheet Boc-Leu-Val-Val-DPro-Gly-Leu-Val-Val-OMe (two conformers in P1, $a=9.739 \AA$, $b=11.519 \AA, c=26.253 \AA, \alpha=98.39, \beta=91.45$ and $\left.\gamma=107.80^{\circ}\right)$; the helix-linker-helix Boc-L-[Val-Ala-Leu-Aib-Val-Ala-Leu]-Acp-D[ValAla-Leu-Aib-Val-Ala-Leu]-OMe (in P4,$a=b=10.094 \AA$, $c=93.383 \AA$ ); and the helix- reverse-helix Boc-L-[Va]-Ala-LeuAib-Val-Ala-Leu]-D-[Val-Ala-Leu-Aib-Val-AlaLeu]OMe (two conformers in $\mathrm{P} 21, \mathrm{a}=10.016 \AA, \mathrm{b}=86.843 \AA, \mathrm{c}=10.036, \beta=90.08^{\circ}$ ). The latter two crystals have very long axes (near $89 \AA$ and $93 \AA$ ), that caused overlap of the diffraction spots; however, the less than ideal data measurements did not hinder the solution process. The data were measured to $0.9 \AA$ resolution. Past experience and more recent experience in direct phase determination, as well as vector search procedures, strongly suggest that data with a resolution of at least $1.1 \AA$ is needed for a successful solution. However, limiting the data used for the Patterson function to a maximum value of $60^{\circ}-65^{\circ}$ for $2 \theta$ (Cu radiation) has usually been necessary for a successful vector search of a fragment. The model used for the search fragment has consisted of $20-25 \%$ of the size of the unknown structure. The model generally contains only backbone and $C \beta$ atoms from the more rigid portion of the known structure due to the inherent flexibility of peptide molecules. It may be desirable, but not necessary, to use a model from a known structure that has a sequence similar to that present in the unknown. Finally, the values of the combined figure of merit for the fit of the search model to the data of the unknown have ranged from 0.2 to 0.9 for successful trials.

The PATSEE program has been used for the above examples.

MS02.03.02 APPLICATIONS OF SnB TO PROTEINS1. RusS Miller, Charles M. Weeks, Hauptman-Woodward Medical Research Institute, 73 High Street, Buffalo, NY USA, (716) 856-9600; miller@hwi.buffalo.edu

$S_{n} B$ is a publicly available direct methods software program based on the Shake-and-Bake method of structure determination. It has been used to solve structures containing as many as 600 nonhydrogen atoms, given data to $1.1 \AA$. This $a b$ initio procedure consists of cyclically processing random structures through a series of phase refinement and Fourier refinement steps in an effort to reduce the value of Hauptman's minimal function.

$\begin{array}{lcccc}\text { Structure } & \begin{array}{c}\text { Nonhydrogen } \\ \text { Atoms }\end{array} & \begin{array}{c}\text { Space } \\ \text { Group }\end{array} & \text { Resolution } & \begin{array}{c}\text { Success } \\ \text { Rate }\end{array} \\ \text { Er-1 pheromone } & 302 & C 2 & 1.00 \AA & 1.5 \% \\ \text { Gramicidin } A & 317 & P 2_{1} 2_{1} 2_{1} & 0.86 \AA & 0.3 \% \\ \text { Crambin } & 400 & P 2_{1} & 0.83 \AA & 4.0 \% \\ \text { Alpha-1 peptide } & 450 & P 1 & 0.93 \AA & 5.0 \% \\ \text { Rubredoxin } & 500 & P 2_{1} & 0.92 \AA & 3.0 \% \\ \text { ToxII } & 620 & P 2_{1} 2_{1} 2_{1} & 0.96 \AA & 1 / 1619\end{array}$

In addition, $S n B$ has also been used to solve numerous previously unknown structures in the 100-200 nonhydrogen atom range, some of which could not be solved by traditional tangent-based direct methods.

Computing platforms for $S n B$ include Unix workstations, the TMC CM-5, the Cray C90, the Cray T3D, and the IBM SP2. It is available at several of the NSF supercomputing centers and has been incorporated into Molecular Structure Corporation's teXsan package, vers. 1.7-3.

This research was supported by NIH grant GM-46733 and NSF grant IRI9412415. 2Percentage of randomly generated trial structures that go to solution.

iStructure initially solved by $\mathrm{SnB}$ : Anderson et al.,Abs. ACA (23), 4a.7.A, p. 80. ${ }^{4}$ Stricture initially solved by SnB: Prive et al., Abs. ACA (23), W008, p. 149.

MS02.03.03 APPLICATION OF DIRECT METHODS IN ELECTRON CRYSTALLOGRAPHY. Fan Hai-fu, Wan Zhenghua and Mo You-de, Institute of Physics, Chinese Academy of Sciences, Beijing 100080, P.R. China

Many important crystalline materials are too small in grain size and too imperfect in periodicity for $\mathrm{X}$-ray single crystal analysis to be carried out, but they are suitable for high resolution electron microscopic observation. The electron microscope is the only instrument that can produce simultaneously for a crystalline sample a micrograph and a diffraction pattern corresponding to atomic resolution. Direct methods have been used for ab initio phasing of electron diffraction data from high-Tc superconductors with incommensurate modulations on their structure. On the other hand direct methods have also been used in the image processing of high resolution electron micrographs, where direct methods play an important role in both the image deconvolution and the resolution enhancement. A program has been written to automate the process. The program runs under Microsoft Windows version 3.1 or higher, in an IBM 386 compatible personal computer or better.

MS02.03.04 CRYSTALSTRUCTURES FROMPOWDERDATA BY DIRECT METHODS. W. Lasocha(1), R. Peschar(2), J. Jansen(2) \& H. Schenk(2) (1)Faculty of Chemistry, Jagiellonian Univ. Krakow, Poland, (2)Lab.v.Kristallografie, Univ.v.Amsterdam, The Netherlands.

In our structural investigations from powder data we used the $\mathrm{Di}$ rect Method Powder Diffraction Package POWSIM[1] to solve BOTH the problem of overlapping reflections and subsequently the crystal structure. Crystal structure detemination from powder diffraction data by means of ' $a b$ initio' methods requires the knowledge of intensities of the majority of the reflections. However, even the best fitting procedure cannot retrieve accurate intensities for reflections whose diffraction angles differ less than some critical value. In POWSIM several relations from Direct Methods, and one relation based on the Patterson function, are employed to predict which overlapping reflections are strong or weak. This information and the sum intensity of overlapping peaks can be used to redistribute the intensity in most of the clusters and to assign statistical weights to the individual decomposed intensities. Using POWSIM we solved stuctures of several fibrillar trimolybdates of silver, potassium, ammonium and anilinum[2], and anilinum pentamolybdate[3]. The most complicated structure in this family of compounds is anilinum trimolybdate with 27 atoms in the asymmetric unit. The intensity-redistribution procedure allowed straightforward location of 18 atoms by the structure solution program SIMPEL (part of POWSIM). The results clearly indicate the potential of the method we used. In our work we use X-ray data collected by a conventional powder diffractometer.

W.L. acknowledges support of the Polish Comm. of Science Res.

1 J. Jansen, R. Peschar, H. Schenk J.Appl. Cryst, 25, 231 (1992), ibid 25, 237(1992) and Z. Kristallog: 206,33(1993)

2 W. Lasocha, J. Jansen, H. Schenk, J. Solid State Chem,109, 1(1994),ibid 115,225(1995),ibid 116,422(1995).ibid 117,(1995)

3 W. Lasocha, H. Schenk, J. Appl. Cryst, submitted 